\title{
Running Characteristics of Plantar Flexor Forces Based on Different Foot Strike Patterns in Medial Tibial Stress Syndrome
}

\author{
Byungjoo Noh ${ }^{*}$
}

${ }^{1}$ Department of Health Care and Science, College of Health Sciences, Dong-A University, Saha-gu, Busan, Republic of Korea

\author{
Received: September 4, 2020 \\ Accepted: October 15, 2020 \\ Published online: October 31, 2020 \\ Keywords: \\ Medial Tibial Stress Syndrome \\ Plantar Flexor Forces \\ Tibial Fascial-Traction Theory
}
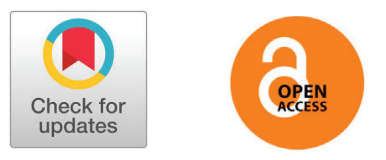

\section{ABSTRACT}

OBJECTIVES The purpose of this study was to compare plantar flexor force due to different foot strike patterns during running in barefoot and shod condition with and without medial tibial stress syndrome (MTSS).

METHODS Fifteen collegiate soccer players who volunteered to participate were divided into MTSS group and controls. Participants' running at $3.3 \mathrm{~m} / \mathrm{s}$ with and without shoes were recorded with a 12-camera system at a sampling frequency of $250 \mathrm{~Hz}$. Each subject completed different foot strike patterns of running as the forefoot strike pattern (FFS) and rearfoot strike pattern (RFS) were collected. Plantar flexor forces were investigated by software for interactive musculoskeletal modeling.

RESULTS Normalized plantar flexor forces in barefoot running with the FFS pattern had greater soleus and peroneus brevis muscle forces in the MTSS group than in controls during the first half of stance, although there were no statistically significant differences for other plantar flexor muscle forces between groups and shod running. In plantar flexor forces due to foot strike pattern, the FFS pattern showed higher plantar flexor forces than the RFS pattern.

CONCLUSIONS The results of musculoskeletal modeling suggest that subjects with MTSS have greater muscle forces of the plantar flexor during running, especially barefoot running with the FFS pattern. It also suggests increased forces in some plantar flexors generated great traction force by repetitively landing on connective tissues in the deep crural fascia causing inflammation at the posteromedial site of the tibia in MTSS.

(c) The Asian Society of Kinesiology and the Korean Academy of Kinesiology

\section{Introduction}

Medial tibial stress syndrome (MTSS) is a common injury among lower extremity running-related injuries [1], which was defined as pain in the posteromedial border of the tibia on palpation of at least $5 \mathrm{~cm}$. Furthermore, patients with MTSS had suffered symptoms for at least 2 weeks [2]. Previous studies have reported that plantar flexors were involved in the development of MTSS as mechanical factors [3-6]. Plantar

*Correspondence: Byungjoo Noh, Department of Health Care and Science, Dong-A University, 37 Nakdong-Daero 550beon-Gil, Saha-gu, Busan, Republic of Korea 49315; Tel: +82-51-200-7515; Fax: +82-51-200-7505; E-mail: bnoh@mtu.edu flexors, especially the soleus, are connected with the deep crural fascia (DCF) attached to the tibia bone, and it is possible to induce traction-induced injury by the DCF [7]. Meanwhile, a well-known theory by Devas [8] has addressed traction to the periosteum by any strong force exerted by calf muscles. This theory was approved as the most apparent etiology of MTSS. However, inadequate anatomical evidence exists to show the development of MTSS, and its causes are still being debated. Hence, more research is needed with musculoskeletal models to determine its mechanisms.

Running is an integral part of most of the sports such as 
soccer, and is associated with an increased running injury risks to the lower extremity [9]. Recently, numerous studies focused on the influence of different foot strike patterns on lower extremity injury during running as one risk factor [10-14]. The foot strike pattern was defined by Cavanagh and Lafortune [15] as the part of the first landing point with the landed surface of the foot as the forefoot strike (FFS) or rearfoot strike (RFS). Numerous studies on the FFS pattern have found a relatively smaller risk of running injury than associated with the RFS pattern [10,12]. Lieberman et al. [10] found that running with the FFS pattern causes comparatively smaller collision forces than the RFS pattern. The shock can be absorbed by the more flexed plantar posture of the ankle joint and more compliance during impact, which decreases the effective body mass that collides with the ground. However, it is possible that there is an increased load to the calf muscles during impact. Previous research also reported that the collision forces are not as important as the acting force in running overuse injuries because the greater magnitudes of internal loads can damage the soft tissues, typically in the lower extremity, more than external loads [16]. Additionally, some studies have recently started to investigate the onset of running injuries in response to the FFS pattern [11, 13-14].

The literature regarding the foot strike pattern still debates which foot strike pattern is better for the reduction of running injuries. In addition, the foot strike pattern associated with the development of MTSS is unclear, so determining the effect of the foot strike pattern on the onset of MTSS during running is required. Notably, plantar flexion muscles play a critical role and may compensate activation against excessive foot pronation on striking when running [17]. Therefore, the acting mechanical characteristics of plantar flexor driven by software for interactive musculoskeletal modeling (SIMM) in athletes with MTSS must be clarified. The purpose of this study was to use a SIMM-driven musculoskeletal model of the lower extremity to estimate plantar flexor forces during running in subjects with MTSS and compare those forces with non-MTSS control subjects who have motion characteristics derived from mechanical factors. We also compared the FFS and RFS patterns in the MTSS group to more clearly establish the mechanism of the development of MTSS. It was hypothesized that when running the plantar flexor forces would be greater in subjects with MTSS than in non-MTSS. We also expected that the greater plantar flexor forces depended on whether the running was done the FFS pattern or the RFS pattern.

\section{Methods}

\section{Subjects}

Two groups of consisted all of the 15 collegiate soccer player subjects were used in the study with the MTSS and without MTSS. Seven of male soccer players with the MTSS separated from subjects with diagnosed the MTSS by an experienced orthopedic surgeon. The clinical diagnosis of the MTSS based on the inclusion criteria [2] as follows: pain in the posteromedial aspect of the tibia caused by exercise or running and palpation pain had been present along the posteromedial border of the tibia at least $5 \mathrm{~cm}$ for at least 2 weeks. The non-MTSS group (control group) was included 8 male soccer players without any pain in the posteromedial aspect of the tibia and without another lower extremity injury. We conducted one-to-one matching between groups on the basis of age, sex, height, total body mass, and BMI for reduction to the effect of their physical characteristics when subjects were recruiting. All methods were performed in accordance with the relevant guidelines and regulations. All participants signed their informed consent after reading all the study details. This study was approved by the Institutional Review Board.

\section{Data Collection}

The participants were given verbal or visual instructions about the FFS and RFS techniques prior to trials. They also were demanded to imagine and practice the techniques before the testing. Each subject was equipped with standardized indoor futsal footwear that was provided to create similar conditions to those of soccer cleats.

Subjects performed each task of running at least three trials of the FFS and RFS at $3.3 \mathrm{~m} / \mathrm{s}$ on a runway (length $5.0 \times$ width $2.5 \mathrm{~m}$ ) in shod condition. Retro-reflective markers were placed on landmarks according to the Plug-in Gait marker set. Three-dimensional marker trajectories measured at a sampling frequency of $250 \mathrm{~Hz}$ using a motion analysis system 
(Vicon MX; Oxford Metrics, Oxford, UK) with 12 cameras (MX T020). After data collection, the marker trajectories data were labeled using the Vicon Nexus software package (Oxford Metrics).

\section{Data Analysis}

This study focused on the stance phase, which was normalized from initial contact (0\%) to toe-off (100\%). The SIMM (Musculographics, Santa Rosa, CA, USA) driven musculoskeletal model by Delp and Loan [18] was used for whole data as muscle forces of the plantar flexor. Each trial was used to determine forces in the muscles of the plantar flexor as follows: the gastrocnemius medialis (GM), gastrocnemius lateralis (GL), soleus (Sol), tibialis posterior (TP), flexor digitorum (FD), flexor hallucis $(\mathrm{FH})$, peroneus brevis $(\mathrm{PB})$, and peroneus longus (PL). Inverse kinematics is used to calculate joint angles by experimentally measured marker positions. Next, calculated joint angles are used to solve the net reaction forces and net moments at each joint by inverse dynamics with angular velocities, angular accelerations, and ground reaction forces. Finally, the estimation of muscle forces is used for the static optimization tool. In the optimization approach, the dynamic equations are solved first to calculate the muscle forces, the net forces and moments at the joints from experimental kinematics measurement (inverse dynamics) [19-20]. The static optimization tool is estimated muscle force by minimizing the sum of the square of muscle excitations while accounting for muscle activation, length, and shortening velocity [21].

This study showed normalized muscle forces of the plantar flexor, which possibly affects the onset of the mechanical factor on the development of MTSS. In order to normalize muscle forces, data measured during running were divided by multiplying the height and body mass for each muscle to facilitate comparison for muscle forces between individuals and groups.

\section{Statistical Analysis}

For all plantar flexor forces from initial contact to toe-off that were calculated by SIMM, two-way factorial analysis of variance was performed to compare the plantar flexor force of each group and foot strike patterns in both conditions.
Subsequently, the two factors which were the groups and the foot strike patterns in both conditions followed by the independent Student's t-test. The statistical analysis was conducted using IBM SPSS Statistics 21 (IBM, Somers, NY, USA). The level of statistical significance was set at 0.05 .

\section{Results}

$<$ Table 1> shows the demographic characteristics of the participants. Comparison of subjects' characteristics between groups that there were no significant differences in demographic characteristics in the two groups.

Table 1. Demographic characteristics of participants

\begin{tabular}{cccc}
\hline Variables & Controls & MTSS & $\mathbf{p}$ \\
\hline Age (years) & $19.6 \pm 1.9$ & $19.8 \pm 1.5$ & 0.80 \\
Height $(\mathrm{m})$ & $1.69 \pm 0.04$ & $1.73 \pm 0.10$ & 0.43 \\
Body mass $(\mathrm{kg})$ & $62.3 \pm 4.4$ & $63.8 \pm 11.1$ & 0.72 \\
BMI $\left(\mathrm{kg} / \mathrm{m}^{2}\right)$ & $21.6 \pm 1.2$ & $21.1 \pm 1.4$ & 0.51 \\
\hline
\end{tabular}

mean $\pm S D$, mean and standard deviation; MTSS, medial tibial stress syndrome; BMI, body mass index; significant difference: $p<0.05$.

\section{Plantar Flexor Forces for Each Group and Foot Strike Pattern}

The means of plantar flexor muscle forces for the entire stance phase during running with and without shoes for different foot strike patterns are shown in $<$ Table $2>$. The main effects were significantly greater $\operatorname{Sol}(\mathrm{F}[1,26]=4.596$, $\mathrm{p}<0.05)$ and $\mathrm{PB}(\mathrm{F}[1,26]=5.580, \mathrm{p}<0.05)$ muscle forces in the barefoot condition in both the MTSS and non-MTSS groups. There were no significant effects for the mean of normalized plantar flexor muscle forces between groups in the shod condition. In the RFS pattern, the MTSS group indicated relatively less plantar flexor forces, or was similar in both groups.

Additionally, significant effects of foot strike pattern were observed for GM (F [1, 26] = 17.020, p < 0.001; in barefoot condition), $(\mathrm{F}[1,26]=4.397, \mathrm{p}<0.05$; in the shod condition), Sol $(\mathrm{F}[1,26]=8.978, \mathrm{p}<0.01$; in the barefoot condition), ( $\mathrm{F}$ $[1,26]=12.135, \mathrm{p}<0.005$; in the shod condition $)$, and TP $(\mathrm{F}$ $[1,26]=5.012, p<0.05$; in the shod condition) muscle forces in the MTSS group. There was a tendency towards greater GL 
Table 2. Normalized muscle force at the whole stance phase at various conditions

\begin{tabular}{|c|c|c|c|c|c|c|c|c|}
\hline \multirow{2}{*}{ Muscles } & \multicolumn{4}{|c|}{ Barefoot condition } & \multicolumn{4}{|c|}{ Shod condition } \\
\hline & CON with FFS & CON with RFS & MTSS with FFS & MTSS with RFS & CON with FFS & CON with RFS & MTSS with FFS & MTSS with RFS \\
\hline Gastrocnemius medialis ${ }^{B}$ & $7.97 \pm 3.09$ & $6.97 \pm 3.03$ & $8.33 \pm 3.80$ & $6.72 \pm 3.37$ & $5.92 \pm 3.24$ & $5.42 \pm 3.49$ & $6.28 \pm 3.55$ & $5.41 \pm 3.52$ \\
\hline Gastrocnemius lateralis ${ }^{\mathrm{B}, \mathrm{S}}$ & $3.75 \pm 0.91$ & $3.57 \pm 0.86$ & $3.92 \pm 1.30$ & $3.48 \pm 0.91$ & $3.75 \pm 0.95$ & $3.22 \pm 0.94$ & $3.30 \pm 1.17$ & $3.12 \pm 1.02$ \\
\hline Soleus ${ }^{B, S, G}$ & $16.56 \pm 15.39$ & $15.29 \pm 14.38$ & $19.91 \pm 17.92$ & $14.08 \pm 14.03$ & $18.11 \pm 15.71$ & $14.56 \pm 13.90$ & $17.39 \pm 17.56$ & $13.30 \pm 13.03$ \\
\hline Tibialis posterior $^{B}$ & $5.27 \pm 2.54$ & $5.48 \pm 2.69$ & $4.61 \pm 2.25$ & $4.97 \pm 2.40$ & $7.74 \pm 4.42$ & $6.45 \pm 4.16$ & $6.67 \pm 4.80$ & $5.56 \pm 3.94$ \\
\hline Flexor digitorum ${ }^{\mathrm{s}}$ & $1.93 \pm 0.30$ & $1.95 \pm 0.34$ & $1.83 \pm 0.33$ & $1.87 \pm 0.35$ & $2.12 \pm 0.49$ & $1.92 \pm 0.45$ & $1.93 \pm 0.52$ & $1.86 \pm 0.46$ \\
\hline Flexor hallucis ${ }^{s}$ & $2.01 \pm 0.30$ & $1.92 \pm 0.34$ & $1.90 \pm 0.36$ & $1.86 \pm 0.36$ & $2.12 \pm 0.51$ & $1.96 \pm 0.46$ & $1.93 \pm 0.52$ & $1.92 \pm 0.47$ \\
\hline Peroneus brevis ${ }^{B, S, G}$ & $2.44 \pm 0.32$ & $2.32 \pm 0.27$ & $2.68 \pm 0.56$ & $2.50 \pm 0.40$ & $2.65 \pm 0.31$ & $2.40 \pm 0.33$ & $2.36 \pm 0.52$ & $2.22 \pm 0.45$ \\
\hline Peroneus longus ${ }^{\mathrm{B}, \mathrm{G}}$ & $6.63 \pm 1.47$ & $6.23 \pm 1.30$ & $6.60 \pm 1.87$ & $6.38 \pm 1.46$ & $5.09 \pm 0.63$ & $4.63 \pm 0.61$ & $4.33 \pm 0.74$ & $4.56 \pm 1.03$ \\
\hline
\end{tabular}

mean \pm SD, mean and standard deviation; CON, controls; MTSS, medial tibial stress syndrome; FFS, forefoot strike; RFS, rearfoot strike; B (barefoot condition) and $\mathrm{S}$ (shod condition) indicates a main effect of the foot strike pattern and $\mathrm{G}$ indicates a main effect of groups in barefoot condition; significant difference, $p<0.05$.

( $p=0.095$; in the barefoot condition, $\mathrm{p}=0.068$; in the shod condition) muscle forces in both the MTSS and non-MTSS groups. There were no significant interactive effects between groups regarding the foot strike pattern.

\section{Comparison of Normalized Plantar Flexor Forces}

In a comparison of groups, Sol (at first 5-40\% of stance) and $\mathrm{PB}$ (at first $40-50 \%$ of stance) muscle forces were significantly greater in the MTSS group than in the non-MTSS group during barefoot running with the FFS pattern $(\mathrm{p}<0.05)$.

Additionally, in a comparison of different foot strike pattern in the barefoot condition, GM (at first 10-50\% of stance), GL (at first 15-55\% of stance), and Sol (at first 5-45\% of stance) muscle forces with the FFS pattern were significantly greater than with the RFS pattern in the MTSS group during the first half of stance $(\mathrm{p}<0.05)$.

In a comparison of different foot strike pattern with shod condition, the GM (at first 15-50\% of stance), GL (at first 15$50 \%$ of stance), Sol (at first 5-50\% of stance), and TP (at first $10-45 \%$ of stance) muscle forces had significantly greater in the FFS pattern than the RFS pattern in the MTSS group ( $\mathrm{p}<$ 0.05). Next, GM (at first 90-100\% of stance) and Sol (at first $70-80 \%$ of stance) muscle forces where significantly greater in the RFS pattern than in the FFS pattern in the MTSS group $(\mathrm{p}<0.05)$.

The MTSS group and the non-MTSS group had broadly similar features in all conditions of running, although more generated plantar flexor forces were observed in the MTSS group than in the non-MTSS group.

\section{Discussion}

Using SIMM, this study investigated the muscle forces that occur in the plantar flexor during running in subjects with MTSS. We then compared these findings with those of uninjured control subjects. The data partially confirmed our hypothesis that subjects with MTSS have greater plantar flexor force than that in control subjects. We also expected greater plantar flexor force with the FFS pattern than with the RFS pattern. Our results showed that a few of the plantar flexors, such as Sol and PB, relatively increased muscle forces could indicate the generation of traction force of certain connective tissues repetitively during the first half of the stance in MTSS. Additionally, the FFS pattern has indicated greater GM, GL, and Sol (in barefoot) and GM, GL, Sol, and TP (in shod) muscle forces during the first half of the stance.

\section{Comparison of Normalized Plantar Flexor Forces between Groups}

We showed that barefoot running with the FFS pattern had greater Sol and PB muscle forces in the MTSS group than in controls during the first half of the stance. Stickley et al. [7] have also reported that the Sol muscle is attached to the tibia connecting with the DCF. Hence, relatively increased Sol muscle forces during the first half of the stance may imply 
that great mechanical stress could be repetitively generated through the DCF causing inflammation at the posteromedial site of the periosteum of the tibia, caused by sudden increased training volume or prolonged running. In shod running, the FFS pattern had tendencies toward greater GM and Sol muscle forces in the MTSS group than in controls during the first half of stance, although there was no statistically significant difference for plantar flexor muscle forces between groups. However, such results may imply important points. Some literature reported that MTSS was caused by comparatively greater plantar flexor forces (or tension) $[6,22]$, even though there was a relatively small difference between groups, repetitive stress could generate traction force on the periosteum of the tibia in subjects with MTSS. Because of the repetitive loading could create microscopic damage in tissue [23]. However, further research is needed to investigate the physiological mechanism of this repetitive stress.

We also assessed the time to reach the peak value of muscle forces. Relatively increased GL (FFS in the barefoot condition) and Sol (FFS in the shod condition) muscle forces in the MTSS group showed a tendency for an earlier peak value than the non-MTSS group. Abnormal traction force may have occurred strongly and rapidly and damaged the soft tissue repetitively during the first half of the stance. However, this needs further verification. The combined action of triceps surae muscles produces $80 \%$ of plantar flexion force associated with inversion/supination. This plantar flexion force is produced in response to the great triceps surae muscle-cross sectional area and relatively longer moment arm length [24], which could apply a great traction force on the periosteum of the tibia.

There were no statistically significant differences for plantar flexor muscle forces between groups in the shod condition, although there were greater tendencies toward force of the GM and Sol muscle forces during running in subjects with MTSS than in the controls. In addition, PB and PL muscle forces in the MTSS group indicated a relatively low level of muscle force compared to controls. We may consider plantar flexor forces in the MTSS group were relatively stabilized in response to footwear. Footwear technology has been developed to prevent lower leg muscle overuse and should provide stability and motion control for the runner [25-26]. Thus, plantar flexor muscle forces in the MTSS group were able to maintain a more stable condition in the shod condition, and footwear may help to decrease the onset of MTSS than barefoot condition.

\section{Comparison of Plantar Flexor Forces in Response to Foot Strike Patterns in MTSS}

Our results showed that the greater GM, GL, and Sol (in barefoot) and GM, GL, Sol, and TP (in shod) muscle forces were determined in the FFS pattern during the first half of the stance. This result is in consensus with a previous study that demonstrated similar results of plantar flexor forces being greater in the FFS pattern compared to the RFS pattern during the first half of the stance [9]. Furthermore, the FFS pattern has a comparatively smaller impact peak of vertical ground reaction force than the RFS pattern during running [10]. In a more plantar flexed posture of the ankle joint when the foot strikes in the running, the plantar flexor contracts eccentrically [27] for the generation of smaller collision force.

Based on these findings, the FFS pattern can generate great traction force than the RFS pattern at initial contact by increased plantar flexor force. This result contrasted with previous studies that reported a reduction in running injury from using the FFS pattern $[10,12]$. Ultimately, these findings suggest that great plantar flexor force in the FFS pattern leads to connective tissues (as the DCF) of the lower extremity as traction force, and the development of MTSS may indicate that increased plantar flexor forces act in the initial contact of running. Rooney and Derrick [9] concluded that it is necessary to improve understanding of the potential for injury in response to different foot strike patterns. Thus, it implies that these results could be a key role for athletes to find and choose their proper foot strike pattern, considering their physical characteristics such as body alignment in order to prevent or reduce running injuries.

However, our results also indicate that the RFS pattern delayed show after the FFS pattern, fundamentally, similar to both foot strike patterns. A previous study reported that relatively higher training loads could lead to the onset of overuse injuries [28]. The results of this study showed that a sharp increase of muscle force acted as a great load on the tibia with repetition, and the amount of time spent training 
or running, as well as this great load, might be combined with other risk factors. Especially, the FFS pattern generates a greater load on plantar flexor than the RFS pattern; therefore, great stress could be applied to the tibia by both small amounts of time spent moving, and repetitive training or running. Further research is needed in order to establish the cause of the development of MTSS in athletes, which may involve their foot strike pattern in running or training and the stress (or load) carrying path in response to different foot strike patterns. Our results provide valuable information that may be used in training feedback for the foot strike pattern for the preventive management of athletes with MTSS.

Several limitations of this study should be noted. Firstly, a previous study [29] investigated the plantar flexors activation during running, and this study showed similar features and patterns. Estimated plantar flexor activation and force were calculated as two times of maximum isometric muscle force of the static model. Muscle force was normalized by multiplying the height and body mass for each muscle. Thus, we might find similar features and patterns between muscle activation and force that occurred in response to this interpretation. However, these results are consistent with literature that suggests similar timing and magnitudes of muscle activation and force by EMG [9, 30-31]. Secondly, the muscle is a solid, not a rigid body. Therefore, the muscle would change due to the position of the joint and contraction and also has several muscle origins and insertions. Additionally, there is a normal anatomic variation for each individual. Furthermore, a simulated musculoskeletal model by optimization is still technically incomplete at present. Currently, the development of the algorithm is being done is a newer version. Despite this, simulated results could be changed by a change of maximum isometric force. For this reason, the traction-induced theory of MTSS could not be verified in this study. Further research is needed to investigate the validity of this simulated model and should consider other methods to verify the traction-induced theory of MTSS.

\section{Conclusions}

This study demonstrated plantar flexor forces compared to subjects with MTSS and uninjured controls in response to different foot strike patterns during barefoot and shod running. Partial hypotheses can be derived from this study are there were greater Sol and PB muscle forces in subjects with MTSS during the first half of stance in the barefoot condition; however, there were no statistically significant differences for other plantar flexor forces between groups and in the shod condition. On the other hand, GM, GL, and Sol (in barefoot) and GM, GL, Sol, and TP (in shod) muscle forces were greater in the FFS pattern than in the RFS pattern during the first half of stance in the MTSS group. This could suggest that abnormal mechanical stress in response to increased muscle force is placed on the posteromedial site of the tibia by repetitive landing in athletes, especially in those who tend to use the FFS pattern during the first half of the stance and suddenly increase training volume or running.

\section{Acknowledgments}

This work was supported by the National Research Foundation of Korea (NRF) grant funded by the Korea government (MSIT) (No. 2018085802).

\section{Conflicts of Interest}

The author declares no conflict of interest.

\section{References}

1. Clanton T, Solcher B. Chronic leg pain in the athlete. Clin Sports Med. 1994; 13(4):743-759.

2. Yates B, White S. The incidence and risk factors in the development of medial tibial stress syndrome among naval recruits. Am J Sports Med. 2004; 32(3):772-780.

3. Jones D, James S. Overuse injuries of the lower extremity: shin splints, iliotibial band friction syndrome, and exertional compartment syndromes. Clin Sports Med. 1987; 6(2):273-290.

4. Saxena A, O'Brien T, Bunce D. Anatomic dissection of the tibialis posterior muscle and its correlation to medial tibial stress syndrome. J Foot Surg. 1989; 29(2):105-108.

5. Beck BR, Osternig LR. Medial tibial stress syndrome. The location of muscles in the leg in relation to symptoms. 
J Bone Jt Surg. 1994; 76(7):1057-1061.

6. Bouche RT, Johnson CH. Medial Tibial Stress Syndrome (Tibial Fasciitis) A Proposed Pathomechanical Model Involving Fascial Traction. J Am Podiatr Med Assoc. 2007; 97(1):31-36.

7. Stickley CD, Hetzler RK, Kimura IF, et al. Crural fascia and muscle origins related to medial tibial stress syndrome symptom location. Med Sci Sports Exerc. 2009; 41(11):1991-1996.

8. Devas M. Stress fractures of the tibia in athletes or "shin soreness". Bone Joint J. 1958; 40(2):227-239.

9. Rooney BD, Derrick TR. Joint contact loading in forefoot and rearfoot strike patterns during running. J Biomech. 2013; 46(13):2201-2206.

10. Lieberman DE, Venkadesan M, Werbel WA, et al. Foot strike patterns and collision forces in habitually barefoot versus shod runners. Nature. 2010; 463(7280):531.

11. Giuliani J, Masini B, Alitz C, et al. Barefoot-simulating footwear associated with metatarsal stress injury in 2 runners. Orthopedics. 2011; 34(7):e320-e323.

12. Daoud AI, Geissler GJ, Wang F, et al. Foot strike and injury rates in endurance runners: a retrospective study. Med Sci Sports Exerc. 2012; 44(7):1325-1334.

13. Salzler MJ, Bluman EM, Noonan S, et al. Injuries observed in minimalist runners. Foot Ankle Int. 2012; 33(4):262266.

14. Olin ED, Gutierrez GM. EMG and tibial shock upon the first attempt at barefoot running. Hum Mov Sci. 2016; 32(2):343-352.

15. Cavanagh PR, Lafortune MA. Ground reaction forces in distance running. J Biomech. 1980; 13(5):397-406.

16. Scott SH, Winter DA. Internal forces of chronic running injury sites. Med Sci Sports Exerc. 1990; 22(3):357-369.

17. Beck BR. Tibial stress injuries. Sports Med. 1998; 26(4):265-279.

18. Delp SL, Loan JP. A graphics-based software system to develop and analyze models of musculoskeletal structures. Comput Biol Med. 1995; 25(1):21-34.

19. Anderson FC, Pandy MG. Dynamic optimization of human walking. J Biomech Eng. 2001; 123(5):381-390.
20. Pandy MG. Simple and complex models for studying muscle function in walking. Philos Trans R Soc Lond B Biol Sci. 2003; 358(1437):1501-1509.

21. Zajac FE. Muscle and tendon: properties, models, scaling, and application to biomechanics and motor control. Crit Rev Biomed Eng. 1989; 17(4):359-411.

22. Detmer DE. Chronic shin splints. Sports Med. 1986; 3(6):436-446.

23. Adams MA. Biomechanics of back pain. Acupunct Med. 2004; 22(4):178-188.

24. Murray MP, Guten GN, Baldwin JM, et al. A comparison of plantar flexion torque with and without the triceps surae. Acta Orthop Scand. 1976; 47(1):122-124.

25. Drez D. Running footwear: examination of the training shoe, the foot, and functional orthotic devices. Am J Sports Med. 1980; 8(2):140-141.

26. Cheung RT, Ng GY, Chen BF. Association of footwear with patellofemoral pain syndrome in runners. Sports Med. 2006; 36(3):199-205.

27. Michael RH, Holder LE. The soleus syndrome: a cause of medial tibial stress (shin splints). Am J Sports Med. 1985; 13(2):87-94.

28. Jones BH, Cowan DN, Tomlinson JP, et al. Epidemiology of injuries associated with physical training among young men in the army. Med Sci Sports Exerc. 1993; 25(2):197-203.

29. Noh B, Ishii T, Masunari A, et al. Muscle activation of plantar flexors in response to different strike patterns during barefoot and shod running in medial tibial stress syndrome. J Phys Fit Sports Med. 2015; 4(1):133-141.

30. Neptune RR, Sasaki K. Ankle plantar flexor force production is an important determinant of the preferred walk-to-run transition speed. J Exp Biol. 2005; 208(5):799-808.

31. Haight DJ, Lerner ZF, Board WJ, et al. A comparison of slow, uphill and fast, level walking on lower extremity biomechanics and tibiofemoral joint loading in obese and nonobese adults. J Orthop Res. 2014; 32(2):324330. 